\title{
Clinical Outcomes of Ankle Distraction Arthroplasty Versus Supramalleolar Osteotomy for Treatment of Post-Traumatic Ankle Arthritis
}

\section{Zongyu Yang}

Third Hospital of Hebei Medical University

Liang Cui

Cangzhou Hospital of Integrated TCM-WM

\section{Shiwu Tao}

Cangzhou Hospital of Integrated TCM-WM

\section{Li Wang}

Third Hospital of Hebei Medical University

\section{Fengqi Zhang}

Third Hospital of Hebei Medical University

Jianyong Zhao

Cangzhou Hospital of Integrated TCM-WM

Xinzhong Shao ( $\nabla$ drtgyx@163.com )

Third Hospital of Hebei Medical University

\section{Research Article}

Keywords: Post-traumatic ankle arthritis, Clinical outcome, Ankle distraction arthroplasty, Supramalleolar osteotomy

Posted Date: January 17th, 2022

DOI: https://doi.org/10.21203/rs.3.rs-1140232/v1

License: () (i) This work is licensed under a Creative Commons Attribution 4.0 International License. Read Full License 


\section{Abstract}

\section{Background}

Post-traumatic ankle arthritis is increasing, especially in young people and currently there is still lack of solid guidelines regarding its operative treatment. Ankle distraction arthroplasty and supramalleolar osteotomy are both options for such medical condition, but the studies comparing their clinical outcomes have been scarce. This study aimed to address this issue.

\section{Methods}

This was designed a retrospective study, enrolling 73 consecutive patients who underwent ankle distraction arthroplasty $(n=32)$ or supramalleolar osteotomy $(n=41)$ for post-traumatic ankle arthritis from January 2015 to December 2018. All patients had at least 24-month follow-ups. The outcome measures were the comparisons of the pre- and postoperative visual analog scale (VAS) for pain relief, the American Orthopedic Foot \& Ankle Society (AOFAS) ankle-hindfoot scores, complications, patient-rated overall satisfaction and ankle function.

\section{Results}

At an average of 32 months (range, 24 to 48 months) follow-up, both groups achieved significantly improved VAS and AOFAS scores compared to those at baseline (all $p<0.05$ ), but not significantly differed between groups (all $p>0.05$ ). The ankle distraction arthroplasty group had better postoperative ankle mobility than the supramalleolar osteotomy group, in terms of plantarflexion ( $37.8 \pm 4.2$ vs $30.4 \pm 3.6, P=0.022)$, dorsiflexion $(36.5 \pm 6.4$ vs $28.3 \pm 5.5, P=0.011)$, varus $(32.1 \pm 4.5$ vs $27.1 \pm 3.1, P=0.029)$ and valgus $(28.4 \pm 3.7$ vs $25.2 \pm 2.8, P=0.047)$. Either, both groups did not differ in in the tibial anterior surface angle, talar tilt angle, tibial lateral surface angle, except for the correcting the load-bearing line of the ankle and hindfoot. The prevalence of postoperative complication was not significantly different between both groups (28.1\% vs $17.1 \%, \mathrm{P}=0.257)$.

\section{Conclusions}

Both ankle distraction arthroplasty and supramalleolar osteotomy are effective treatment options for Takakura-Tanaka stage 3 post-traumatic ankle arthritis, but with respective advantages and disadvantages. In practice, individualized treatment option tailored to the medical conditions and patients' specific need should be considered before a decision is made.

\section{Introduction}

Ankle osteoarthritis is an important cause of ankle pain and functional limitation, despite the lower incidence rate than knee and hip arthritis [1]. From the anatomic view, ankle site had approximately $1 / 2$ to $3 / 5$ the maximum cartilage thickness at weight-bearing areas as that of hip and knee $(2.7 \mathrm{~mm}$ vs $3-6 \mathrm{~mm})$ [2]. Thus, any abnormal alignment of the ankle would lead to local stress concentration and accelerate the degeneration of ankle cartilage and the formation of ankle arthritis [3]. It has been estimated that $20-40 \%$ of patients with acute ankle trauma like ankle sprain would develop chronic sprain, leading injury or even rupture of the lateral ligaments of the ankle, causing chronic lateral ankle instability and the secondary post-traumatic ankle arthritis [4]. The recent reports showed that the young patients were the predominant for this arthropathy and the incidence seemed on the rise $[5,6]$. Currently, there is well-established consensus that ankle arthrodesis is the standard treatment for the end-stage traumatic ankle arthritis (stage 4 defined by Takakura-Tanaka classification), which typically presents with persistent swelling around the ankle with pain, loss of normal bone structure of the tibiotalar joint surface and basic loss of function $[7,8]$. Also, for stage 1 and 2 ankle 
arthritis, arthroscopic surgery or open joint debridement has been considered as the standard treatment, and the reported clinical outcome are confirmative and accepted for the majority of patients. However, for stage 3 ankle arthritis, there is lack of substantial evidence on the surgical option when decision-making is considered. The most commonly used surgical treatment methods were ankle distraction arthroplasty and supramalleolar osteotomy, with respective advantages and disadvantages. The ankle distraction arthroplasty could achieve the early functional outcomes, but still was associated with relatively high failure rate, especially for those obese patients and those with large talar tile angles $[2,9]$; in contract, supramalleolar osteotomy is advantageous in correcting the load-bearing line of the ankle and hindfoot and having fewer complications, but is limited by the inability to achieve a fast recovery [1]. By far as we know, there are no studies directly comparing the outcomes of both surgical methods for stage 3 ankle arthritis.

Considering the increasing importance of evidence-based data on decision-making for the surgical option for this increasingly growing arthropathy in the young patients, it is necessary to clarify the respective advantages and disadvantages of surgical methods to facilitate the effective management. Given that, we designed this study, with aims to compare the clinical outcomes between ankle distraction arthroplasty and supramalleolar osteotomy for stage 3 ankle arthritis, in terms of pain relief, functional recovery and postoperative complications.

\section{Materials And Methods}

This was a retrospective study. The study protocol was approved by the institutional review board of Cangzhou Hospital of Integrated TCM-WM and all patients provided the written informed consent.

The inclusion criteria were age $>18$ years; stage 3 post-traumatic ankle arthritis classified as Takakura-Tanaka classification, ankle pain and swelling lasting $>3$ months and failure of conservative treatment; absence of past any surgical procedure around the ankle; complete pre- and postoperative data and imaging examinations, and the followup of at least 24 months.

The exclusion criteria were recent infection around the ankle; history of ankle fracture; other serious deformities or diseases of the foot and ankle, such as clubfoot or diabetic foot; incomplete medical record data or lost to follow-up or follow-up period < 24 months. Additionally, surgery is contraindicated for those with congenital collagen deficiency; bodyweight >120 kg; severe heart disease; lesions affecting liver and kidney function; severe diabetes; central nervous system diseases; or others.

The above criteria identified 73 consecutive patients who underwent either ankle distraction arthroplasty $(n=32)$ or supramalleolar osteotomy $(n=41)$ for treatment of Takakura-Tanaka stage 3 post-traumatic ankle arthritis after failure of conservative treatment from January 2015 to December 2018.

Among ankle distraction arthroplasty group, there were 19 men and 13 women, with an average age of $54.7 \pm 12.8$ years; left ankle was affected in 14 and right in 18 patients; 21 had a clear history of ankle trauma and 11 with unknown etiology. Among supramalleolar osteotomy group, 27 were men and 14 were women, with an average of $56.4 \pm 11.7$ years; left ankle was affected in 17 and right in 24 patients; 29 had a clear history of ankle trauma and 12 with unknown etiology.

\section{Preoperative examination}

Preoperative evaluation of all patients included a detailed history ankle arthritis, presence of comorbidities, physical examination, and imaging examination, based on which the ankle anteroposterior position, tibial anterior surface angle (TAS), talar tilt angle (TT) and tibial lateral surface angle (TLS) were measured. The calcaneal axial X-ray was taken to assess the force line of the lower limb and evaluate the presence of varus or valgus of the calcaneus and talus; CT was 
performed to evaluate the condition of the subtalar and tibiotalar joints; MRI was performed to evaluate the cartilage condition of the ankle, presence or absence of talar necrosis, condition of the surrounding soft tissue, presence or absence of edema of the surrounding ligaments, and completeness of the lateral ligaments.

\section{Operative procedures}

The supramalleolar osteotomy group received prophylactic intravenous antibiotics 30 minutes prior to skin incision. After anesthetic induction, the patient was placed in supine position then a tourniquet was placed at the proximal extremity. A 4-cm longitudinal incision was made in the middle of the anterior ankle to expose the ankle joint cavity and assess whether there was contact between the ankle and tibial joint surfaces. Intraoperatively, lip-like hyperplasia of the tibial joint surface and hyperplasia of the lateral talar surface can be observed, and the passive movement of the ankle was limited. The proliferative bone was removed, and the ankle was moved passively until the range of motion was close to normal. The joint cavity was washed with normal saline and the surgical incision was sutured. A Kirschner wire was used as an osteotomy guide 4-5 cm above the ankle joint. After the osteotomy direction was confirmed on X-ray fluoroscopy, the medial, anterior, and posterior cortices were cut from the anterolateral side parallel to the articular surface of the distal tibia, and the contralateral cortices and periosteum were retained to form a hinge when the osteotomy was opened and were inserted into the wedge-shaped bone block to increase the stability. After satisfactory correction of the varus deformity, a Kirschner wire was used for temporary fixation. Autogenous or allogeneic bone was implanted at the osteotomy site. An anatomical steel plate was used to fix the osteotomy end and afterwards the incision was closed.

The ankle distraction arthroplasty group underwent the same preoperative preparation and the same ankle debridement procedure as the supramalleolar osteotomy group. The ankle joint was placed in a neutral position and the annular external fixator was placed in a suitable position with the extension rod directly opposite the ankle joint activity center. A 2.0-mm-diameter Kirschner wire was used to drill $8 \mathrm{~cm}$ below the knee joint, and then two Kirschner wires were drilled 5 $\mathrm{cm}$ above the ankle joint and parallel to the knee joint; one Kirschner wire was fixed in front of the calcaneal tubercle, and the other one was fixed in the metatarsal base of the anterior foot. Each ring was reinforced with a threaded needle.

\section{Postoperative management}

The incision was routinely dressed and intravenous prophylactic antibiotics were administered. The Kirschner wire hole was wiped with iodophor every day to prevent sinus tract infection. To promote functional recovery of the ankle and prevent postoperative stiffness, both groups began early postoperative rehabilitation exercises. From postoperative day 1 , patients were instructed to exercise the toes and quadriceps femoris to prevent lower-extremities deep venous thrombosis. At 1 week postoperatively, the ankle was radiographed from the anteroposterior and lateral aspects. In the ankle distraction arthroplasty group, the external fixator was adjusted as needed; the ankle joint cavity was gradually stretched by about $0.5 \mathrm{~mm}$ every day and adjusted every 12 hours until the ankle joint space was pulled out by $5 \mathrm{~mm}$ (the external fixator was adjusted at any time based on the patient's condition). The ankle was half loaded by 1 month postoperatively, and full loaded by 2 months postoperatively. At 3 months postoperatively, the external fixator was removed and ankle rehabilitation training was commenced. The supramalleolar osteotomy group performed the same functional exercises to prevent postoperative ankle stiffness and enhance the joint range of motion.

\section{Outcome Measures}

All patients were assessed by independent investigator preoperatively and at 6 and 24 months postoperatively. Routine radiological examination comprised anteroposterior and lateral radiographs of the ankle, and full-length lower extremity weight-bearing radiographs. A goniometer was used to measure the ankle angle (TAS, TT, and TLS) and compare them between preoperative and postoperative recorded values. 
The range of motion of the affected foot and the healthy foot was measured, including the ranges of varus, valgus, dorsiflexion, and plantarflexion. The American Orthopedic Foot \& Ankle Society (AOFAS) ankle-hindfoot score was used to objectively evaluate the pain, ankle function, gait, and force line of the affected ankle and was rated as excellent (90100 points), good (75-89 points), fair (50-74 points), or poor (0-50 points). Visual analog scales (VAS) scores for used for evaluate pain.

At the last visit, patients were asked to rate their overall satisfaction with their surgical results as excellent, good, fair, or poor. (Table 1)

Table 1 Clinical Rating Scale for Postoperative Ankle function.

\begin{tabular}{|ll|}
\hline Rating & Description \\
\hline Excellent & Full range of motion equal to the contralateral ankle without pain. Un-restricted work or sports activity. \\
\hline Fair & $\begin{array}{l}\text { Functional range of motion and stable ankle. Able to return to the previous level with minimal pain with } \\
\text { work or sport activity }\end{array}$ \\
\hline Poor & Paily living and sports activity. \\
\hline
\end{tabular}

To reduce errors and ensure the accuracy of data, all measurements were independently performed by three investigators, and the average of the three results for any measurement was used in the analysis.

\section{Results}

The average follow-up duration was 32 months, ranging from 24 months to 48 months. There were no significant differences between the two groups regarding age, sex, Takakura-Tanaka stage (all patients had stage 3 ankle arthritis), and follow-up duration (all P > 0.05). (Table 2) 
Table 2

Comparison of the data of patients with ankle distraction arthroplasty and supramalleolar osteotomy.

\begin{tabular}{|c|c|c|c|}
\hline & $\begin{array}{l}\text { Ankle Distraction Arthroplasty ( } \\
\text { and \%, Or mean } \pm \text { SD) }\end{array}$ & $\begin{array}{l}\text { Supramalleolar Osteotomy ( } \\
\text { and \%, Or mean } \pm \text { SD) }\end{array}$ & $p$ \\
\hline Age & $54.7 \pm 12.8$ & $56.4 \pm 11.7$ & 0.801 \\
\hline Gender & & & 0.569 \\
\hline Male & 59.4 & 65.9 & \\
\hline female & 40.6 & 34.1 & \\
\hline Side & & & 0.402 \\
\hline Left & 43.8 & 34.1 & \\
\hline Right & 56.3 & 65.9 & \\
\hline Body mass index $\mathrm{kg} / \mathrm{m}^{2}$ & $28.3 \pm 3.4$ & $29.1 \pm 4.3$ & 0.540 \\
\hline $\begin{array}{l}\text { Time interval between onset } \\
\text { and operation (years) }\end{array}$ & $3.2 \pm 4.3$ & $4.1 \pm 3.5$ & 0.244 \\
\hline Operation time (minutes) & $78 \pm 32$ & $94 \pm 28$ & $<0.001$ \\
\hline Follow up time (month) & $30.8 \pm 5.5$ & $32.9 \pm 7.4$ & 0.601 \\
\hline
\end{tabular}

Among ankle distraction arthroplasty group, the extent of satisfaction was rated as excellent by 15 (46.9\%) patients, good by $10(31.3 \%)$, fair by $5(15.6 \%)$, and poor by $2(6.3 \%)$. Among supramalleolar osteotomy group, the satisfaction was rated as excellent by $20(48.8 \%)$ patients, good by $15(36.6 \%)$, fair by $4(9.8 \%)$ and poor by $2(4.9 \%)$. The excellent and good rate was not significantly different between two groups ( $78.2 \%$ versus $85.4 \%, p=0.422)$.

At final follow-up, the ankle distraction arthroplasty group had significantly better ankle mobility range in terms of varus, valgus, dorsiflexion, and plantarflexion than did the supramalleolar osteotomy group (all $\mathrm{P}<0.05$ ) (Table 3 ). There were no significant differences regarding the final postoperative imaging parameters of TAS ( $92.1 \pm 3.9$ vs $86.2 \pm 4.1, P=0.074)$, TT ( $2.8 \pm 2.0$ vs $3.2 \pm 1.3)$ and TLS ( $82.1 \pm 6.5$ vs $81.2 \pm 2.5, P=0.524)$ (Table 4); but supramalleolar osteotomy performed significantly better than ankle distraction arthroplasty in correcting talus varus deformity and restoring lower limb alignment, namely the absolute difference between postoperative and preoperative parameters (TAS, $11.9 \pm 3.6 \mathrm{vs}$ $1.5 \pm 2.2, \mathrm{P}<0.001 ; \mathrm{TT}, 3.7 \pm 2.3$ vs $1.3 \pm 1.6, \mathrm{P}=0.003 ; \mathrm{TLS}, 5.9 \pm 3.3$ vs $2.8 \pm 1.9, \mathrm{P}=0.001)$. The ankle distraction arthroplasty had a similar mean AOFAS score (85.8 \pm 6.4$)$ and VAS pain score (1.4 \pm 1.3$)$ as the supramalleolar osteotomy group (AOFAS score 88.2 \pm 7.2 , VAS pain score 1.1 \pm 1.4 ). The mean AOFAS and VAS pain scores were significantly improved postoperatively compared with preoperatively in both groups (both $\mathrm{P}<0.001$ ). (Table 5 ) 
Table 3

Comparison of ankle range of motion between the two groups at the last follow-up.

\begin{tabular}{|c|c|c|c|c|c|c|}
\hline & \multicolumn{2}{|c|}{ Ankle Distraction Arthroplasty } & \multicolumn{2}{|c|}{ Supramalleolar Osteotomy } & \multirow[t]{2}{*}{$t^{*}$} & \multirow[t]{2}{*}{$P^{*}$} \\
\hline & Preoperative & Postoperative & Preoperative & Postoperative & & \\
\hline Plantarflexion $\left(^{\circ}\right)$ & $23.3 \pm 3.7$ & $37.8 \pm 4.2$ & $25.1 \pm 4.8$ & $30.4 \pm 3.6$ & 4.318 & 0.022 \\
\hline Dorsiflexion $\left({ }^{\circ}\right)$ & $17.5 \pm 5.8$ & $36.5 \pm 6.4$ & $23.8 \pm 6.1$ & $28.3 \pm 5.5$ & 4.976 & 0.011 \\
\hline Varus $\left({ }^{\circ}\right)$ & $23.6 \pm 6.0$ & $32.1 \pm 4.5$ & $22.7 \pm 4.2$ & $27.1 \pm 3.1$ & 3.693 & 0.029 \\
\hline Valgus $\left({ }^{\circ}\right)$ & $19.8 \pm 4.1$ & $28.4 \pm 3.7$ & $20.0 \pm 3.4$ & $25.2 \pm 2.8$ & 2.686 & 0.047 \\
\hline
\end{tabular}

Table 4

Comparison of imaging angles between the two groups of patients.

\begin{tabular}{|c|c|c|c|c|c|c|}
\hline & \multicolumn{2}{|c|}{ Ankle Distraction Arthroplasty } & \multicolumn{2}{|c|}{ Supramalleolar Osteotomy } & \multirow[t]{2}{*}{$*_{\mathrm{t}}$} & \multirow[t]{2}{*}{${ }^{*} p$} \\
\hline & Preoperative & Postoperative & Preoperative & Postoperative & & \\
\hline $\operatorname{TAS}\left({ }^{\circ}\right)$ & $84.7 \pm 5.7$ & $86.2 \pm 4.1$ & $80.2 \pm 4.6$ & $92.1 \pm 3.9$ & 1.063 & 0.074 \\
\hline TT $\left(^{\circ}\right)$ & $4.5 \pm 2.1$ & $3.2 \pm 1.3$ & $6.3 \pm 3.4$ & $2.8 \pm 2.0$ & 0.544 & 0.161 \\
\hline $\operatorname{TLS}\left({ }^{\circ}\right)$ & $78.4 \pm 3.9$ & $81.2 \pm 2.5$ & $76.2 \pm 5.8$ & $82.1 \pm 6.5$ & 0.138 & 0.524 \\
\hline
\end{tabular}

Table 5

Comparison of clinical score between the preoperative and postoperative functional score among two groups.

\begin{tabular}{|lllllllll|}
\hline & \multicolumn{3}{l}{ Ankle Distraction Arthroplasty } & & \multicolumn{5}{c|}{ Supramalleolar Osteotomy } \\
\cline { 2 - 8 } & Preoperative & Postoperative & $\mathbf{t}$ & $\mathbf{p}$ & Preoperative & Postoperative & $\mathbf{t}$ & $\mathbf{p}$ \\
\hline Pain & $21.7 \pm 6.2$ & $33.4 \pm 3.1$ & -3.318 & 0.021 & $22.4 \pm 5.8$ & $34.7 \pm 3.7$ & -2.920 & 0.015 \\
\hline Function & $19.7 \pm 7.8$ & $42.1 \pm 4.7$ & -3.890 & 0.004 & $19.2 \pm 6.9$ & $42.9 \pm 6.1$ & -6.425 & 0.002 \\
\hline $\begin{array}{l}\text { Force } \\
\text { Line }\end{array}$ & $5.7 \pm 2.8$ & $9.1 \pm 0.8$ & -5.353 & 0.001 & $3.8 \pm 3.4$ & $8.9 \pm 1.1$ & -4.041 & 0.007 \\
$\begin{array}{l}\text { Total } \\
\text { (AOFAS) }\end{array}$ & $50.7 \pm 8.9$ & $85.8 \pm 6.4$ & -8.112 & $<0.001$ & $48.3 \pm 5.7$ & $88.2 \pm 7.2$ & -10.389 & $<0.001$ \\
\hline VAS & $5.6 \pm 1.4$ & $1.4 \pm 1.3$ & -6.221 & 0.001 & $5.8 \pm 1.2$ & $1.1 \pm 1.4$ & -7.261 & $<0.001$ \\
\hline Note, we did not present the between-group results for every item, because they were all not statistically significant. \\
\hline
\end{tabular}

No significant difference for complication prevalence rate was found between both groups ( $28.1 \%$ vs $17.1 \%, P=0.257)$. There were nine complications in the ankle distraction arthroplasty group, including sinus infection of the Kirschner wire and exudation of secretions in four patients, persistent chronic ankle pain in one patient who finally underwent ankle arthrodesis at 31 months after the index operation, readjustment of the external fixator due to an accident in one patient, and ankle stiffness in three patients in whom the ankle motion improved substantially after rehabilitation training. There were seven complications in the supramalleolar osteotomy group, including 3 cases of scar contracture, 
2 cases of superficial surgical incision which later resolved by oral antibiotics, and 2 cases of delayed healing at the osteotomy site which were treated by extracorporeal shock wave and resolved.

\section{Discussion}

Post-traumatic ankle arthritis is very common in clinical practice and is increasingly affecting younger patients [5]. Various surgical treatment regimens have been used in clinical practice [8, 10-14], but the optimal option remains unclarified. The present study retrospectively analyzed 73 patients with Takakura-Tanaka stage 3 post-traumatic ankle arthritis treated with either ankle distraction arthroplasty or supramalleolar osteotomy. The results suggest that ankle distraction arthroplasty was advantageous in restoring the ankle joint space and ankle mobility, while supramalleolar osteotomy performed better in correcting ankle varus deformity and restoring the lower limb alignment. However, in terms of overall ankle function recovery, subjective satisfaction and postoperative complications, no significant differences were observed.

As a classical method, ankle distraction arthroplasty could reduce the abnormal mechanical stress of the ankle through physical means, promote the intermittent flow of synovial fluid in the joint and therefore reduce the ankle joint. Ankle distraction arthroplasty is mainly indicated for ankles with good alignment and peripheral pain and can be a pretreatment before ankle fusion or replacement surgery in young patients. This procedure has proved to be effective in relieving pain and restoring the ankle function [15-19]; Marijinissen et al [9] followed up 11 patients for at least 2 years after arthroplasty and reported significant improvements in pain and functional scores, suggesting that arthroplasty significantly relieve pain, preserve joint range of motion, and delay or reverse trauma. However, the relatively low effective rate, inconvenience and longer period of treatment may limit its more extensive use in practice [20]. Additionally, the gradually lowering postoperative satisfaction over time should be considered and for patients with obvious ankle valgus deformity, ankle joint distraction arthroplasty alone cannot correct the deformity.

In the present study, we did not find the significant difference in overall satisfaction rate, but a tendency towards lower value in those treated by ankle distraction arthroplasty (excellent and good rate of $78.2 \%$ versus $85.4 \%$ for supramalleolar osteotomy). This may be caused by the higher rate of complications associated with ankle distraction arthroplasty, including sinus tract infection, fixation failure, difficulty in moving after surgery, and the need for frequent reviews and external fixation adjustments [15].

The superiority of supramalleolar osteotomy over distraction arthroplasty is the ability to correct the load line of the ankle and hindfoot and to correct the distal tibial deformity in the coronal and sagittal planes[1, 21]. Supramalleolar osteotomy could transfer the stress to the cartilage area that is normal or not been seriously degraded by the means of adjusting the force line of the tibia. In this study, supramalleolar osteotomy proved to better correct talus varus deformity and restore the lower limb alignment, consistent with the previous findings [22, 23], therefore, facilitating delaying the further development of ankle arthritis. Of note, patients with chronic ankle instability caused by severe injury or repeated multiple injuries may develop increased stress in the asymmetric joint spaces, forming painful asymmetric ankle arthritis and ankle point mismatch [2], thus additionally requiring osteotomy surgery to restore the lateral stability [24].

The second advantage of supramalleolar osteotomy is the relatively lower rate of postoperative complications, with postoperative ankle stiffness as the primary one. Others reported complications involved bone union issues at the osteotomy site, and surgical wound related issues, such as dehiscence and infection. In the present study, we reported an average overall complicate rate of $17.0 \%[25,26]$. Despite the above-mentioned advantages, supramalleolar osteotomy was not overwhelmingly recommended, partly due to the need for a second operation to remove the internal fixation material. 
This study suffered from several limitations. First, the retrospective design might impede the accuracy and precise in collecting the data and these data cannot be verified. The use of average values for some outcome measures conducted by 3 independent investigators might compensate for this limitation. Second, due to the limited use in our institution, we included only 73 eligible patients for data analysis, making the comparison not definitely conclusive. It is possible the true differences for some outcome variables are withheld to detect by such limited sample, namely the type

$\nabla$ statistical error. Third, these operative procedures were completed by general orthopaedic surgeons $(n=7)$ and foot and ankle surgeons $(n=4)$, in whom the experience can be different, thus affecting the results. Also, due to the very limited number of procedures for a certain surgeon, we could not make a direct comparison between them. Fourth, this is a single-certain study, limiting the generalizability of our results to other settings. The well-designed large sample studies are warranted to verify our results and to provide a robust evidence for improving the management of this chronic bone condition.

\section{Conclusions}

This study demonstrated that both ankle distraction arthroplasty and supramalleolar osteotomy are effective treatment methods for Takakura-Tanaka stage 3 post-traumatic ankle arthritis, but with respective advantages and disadvantages. Ankle distraction arthroplasty was advantageous in restoring the ankle joint space and ankle mobility, while supramalleolar osteotomy performed better in correcting ankle varus deformity and restoring the lower limb alignment. In practice, individualized treatment option tailored to the medical conditions and patients' specific need should be made when a decision is considered.

\section{Declarations}

All methods were performed in accordance with the relevant guidelines and regulations.

\section{Ethics approval and consent to participate}

All authors confirm that this work was approved by our Institutional Review Board with ethical principles of research. All data follow the declaration of Helsinki.

This work was performed at the Third Hospital of Hebei Medical University, Hebei, China.

All participants signed informed consent.

\section{Consent for publication}

This work was performed at the Third Hospital of Hebei Medical University, Hebei, China.

Availability of data and materials

The patients' data were collected in the Third Hospital of Hebei Medical University. The data sets used and/or analyzed during the current study are available from the corresponding author on reasonable request.

\section{Competing interests}

The authors declare that they have no competing interests.

\section{Funding}

No funding 


\section{Author contributions}

Xinzhong Shao designed the study; Liang Cui and Shiwu Tao and Jianyong Zhao searched relevant literature and analyzed; Li Wang and Fengqi Zhang interpreted the data; Zongyu Yang wrote the manuscript, and Xinzhong Shao approved the final version of the manuscript.

\section{Acknowledgment}

1.We thank Kelly Zammit, from Liwen Bianji (www.liwenbianji.cn/ac) for editing the English text of a draft of this manuscript.

\section{Authors' information}

1. The Third Hospital of Hebei Medical University

2. Cangzhou Hospital of Integrated TCM-WM

\section{References}

1. Lacorda J, Jung H,Im J. Supramalleolar Distal Tibiofibular Osteotomy for Medial Ankle Osteoarthritis: Current Concepts. Clinics in orthopedic surgery. 2020; 12: 271-278.

2. Zhao H, Qu W, Li Y, Liang X, Ning N, Zhang Y, et al. Functional analysis of distraction arthroplasty in the treatment of ankle osteoarthritis. Journal of orthopaedic surgery and research. 2017; 12: 18.

3. Jantzen C, Ebskov L, Andersen K, Benyahia M, Rasmussen P,Johansen J. [Ankle arthrosis]. Ugeskrift for laeger. 2020; 182:

4. Sun Y, Wang H, Tang Y, Zhao H, Qin S,Zhang F. Reconstruction of the lateral ankle ligaments using the anterior half of peroneus longus tendon graft. Foot and ankle surgery: official journal of the European Society of Foot and Ankle Surgeons. 2019; 25: 242-246.

5. Giannini S, Buda R, Faldini C, Vannini F, Romagnoli M, Grandi G, et al. The treatment of severe posttraumatic arthritis of the ankle joint. The Journal of bone and joint surgery. American volume. 2007; 15-28.

6. Hubbard T,Hertel J. Mechanical contributions to chronic lateral ankle instability. Sports medicine (Auckland, N.Z.). 2006; 36: 263-77.

7. Glazebrook M, Daniels T, Younger A, Foote C, Penner M, Wing K, et al. Comparison of health-related quality of life between patients with end-stage ankle and hip arthrosis. The Journal of bone and joint surgery. American volume. 2008; 90: 499-505.

8. Ma N, Li Z, Li D, Hu Y,Sun N. Clinical evaluation of arthrodesis with llizarov external fixator for the treatment of endstage ankle osteoarthritis: A retrospective study. Medicine. 2020; 99: e23921.

9. Marijnissen A, Hoekstra M, Pré B, van Roermund P, van Melkebeek J, Amendola A, et al. Patient characteristics as predictors of clinical outcome of distraction in treatment of severe ankle osteoarthritis. Journal of orthopaedic research: official publication of the Orthopaedic Research Society. 2014; 32: 96-101.

10. Kim J, Suh D, Choi G, Koo B,Kim S. Change in the weight-bearing line ratio of the ankle joint and ankle joint line orientation after knee arthroplasty and high tibial osteotomy in patients with genu varum deformity. International orthopaedics. 2021; 45: 117-124.

11. Lindsey B, Hundal R, Bakshi N, Holmes J, Talusan P,Walton D. Ankle Arthrodesis Through an Anterior Approach. Journal of orthopaedic trauma. 2020; S42-S43. 
12. Liu X, Chang F, Zhang H, Zhong Z, Xue P,Huang B. Ankle distraction arthroplasty for the treatment of severe ankle arthritis: Case report, technical note, and literature review. Medicine. 2020; 99: e22330.

13. Woo B, Lai M, Ng S, Rikhraj I,Koo K. Clinical outcomes comparing arthroscopic vs open ankle arthrodesis. Foot and ankle surgery: official journal of the European Society of Foot and Ankle Surgeons. 2020; 26: 530-534.

14. Zeininger A, Schmitt D, Hughes-Oliver C,Queen R. The effect of ankle osteoarthritis and total ankle arthroplasty on center of pressure position. Journal of orthopaedic research: official publication of the Orthopaedic Research Society. 2021; 39: 1245-1252.

15. Bernstein M, Reidler J, Fragomen A,Rozbruch S. Ankle Distraction Arthroplasty: Indications, Technique, and Outcomes. The Journal of the American Academy of Orthopaedic Surgeons. 2017; 25: 89-99.

16. Gianakos A, Haring R, Shimozono Y, Fragomen A,Kennedy J. Effect of Microfracture on Functional Outcomes and Subchondral Sclerosis Following Distraction Arthroplasty of the Ankle Joint. Foot \& ankle international. 2020; 41: 631-638.

17. Ploegmakers J, van Roermund P, van Melkebeek J, Lammens J, Bijlsma J, Lafeber F, et al. Prolonged clinical benefit from joint distraction in the treatment of ankle osteoarthritis. Osteoarthritis and cartilage. 2005; 13: 582-8.

18. Smith N, Beaman D, Rozbruch S,Glazebrook M. Evidence-based indications for distraction ankle arthroplasty. Foot \& ankle international. 2012; 33: 632-6.

19. van Valburg A, van Roermund $P$, Marijnissen A, van Melkebeek J, Lammens J, Verbout A, et al. Joint distraction in treatment of osteoarthritis: a two-year follow-up of the ankle. Osteoarthritis and cartilage. 1999; 7: 474-9.

20. Nguyen M, Pedersen D, Gao Y, Saltzman C,Amendola A. Intermediate-term follow-up after ankle distraction for treatment of end-stage osteoarthritis. The Journal of bone and joint surgery. American volume. 2015; 97: 590-6.

21. Choi J, Kim K,Suh J. Low Tibial Valgization Osteotomy for More Severe Varus Ankle Arthritis. Foot \& ankle international. 2020; 41: 1122-1132.

22. Deleu P, Naaim A, Chèze L, Dumas R, Devos Bevernage B, Goubau L, et al. The effect of ankle and hindfoot malalignment on foot mechanics in patients suffering from post-traumatic ankle osteoarthritis. Clinical biomechanics (Bristol, Avon). 2021; 81: 105239.

23. Wu Y. [Treatment options of ankle osteoarthritis by stage]. Zhonghua yi xue za zhi. 2019; 99: 1608-1610.

24. Lee H, Wapner K, Park S, Kim J, Lee D,Sohn D. Ligament reconstruction and calcaneal osteotomy for osteoarthritis of the ankle. Foot \& ankle international. 2009; 30: 475-80.

25. Stamatis E, Cooper P,Myerson M. Supramalleolar osteotomy for the treatment of distal tibial angular deformities and arthritis of the ankle joint. Foot \& ankle international. 2003; 24: 754-64.

26. Zhao H L J, Zhang Y. Clinical application of different types supramalleolar osteotomy for varus ankle osteoarthritis. Chin J Anat Clin. 2009; 24: 112-117.

\section{Figures}




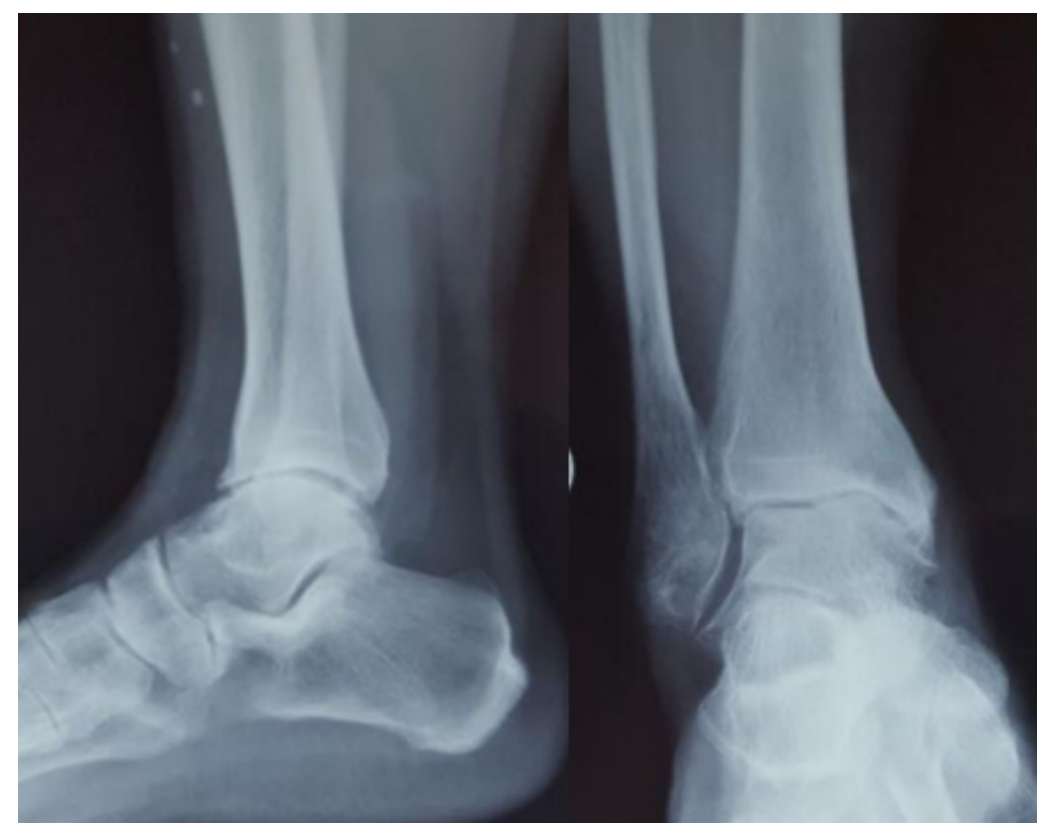

\section{Figure 1}

Preoperative ankle joints in anteroposterior and lateral positions; The space of ankle joint was narrow, osteophytes were found in the anterior tibia and dorsal talus articular surface, the medial and lateral malleolus became sharp, the articular surface of talar dome was inclined to the medial side of the distal tibia, and the ankle was slightly varus.

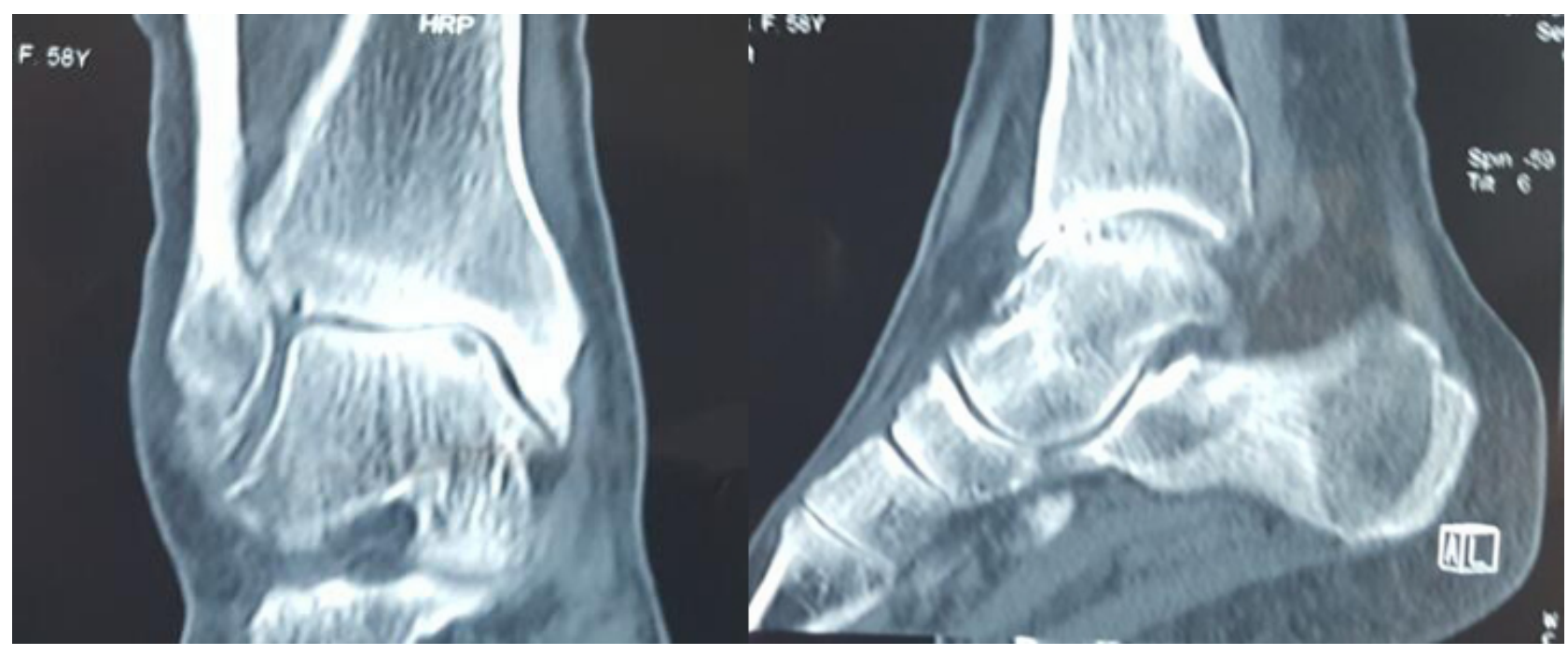

\section{Figure 2}

CT coronal and sagittal images of ankle joint showed that the space of ankle joint was narrow, the osteophyte of anterior tibia was proliferated obviously, the space of medial ankle joint was occluded, the contact of subchondral bone was limited to the medial side, the bone of medial talus was sclerotic and cystic changes appeared, and the medial malleolus became sharp obviously. 


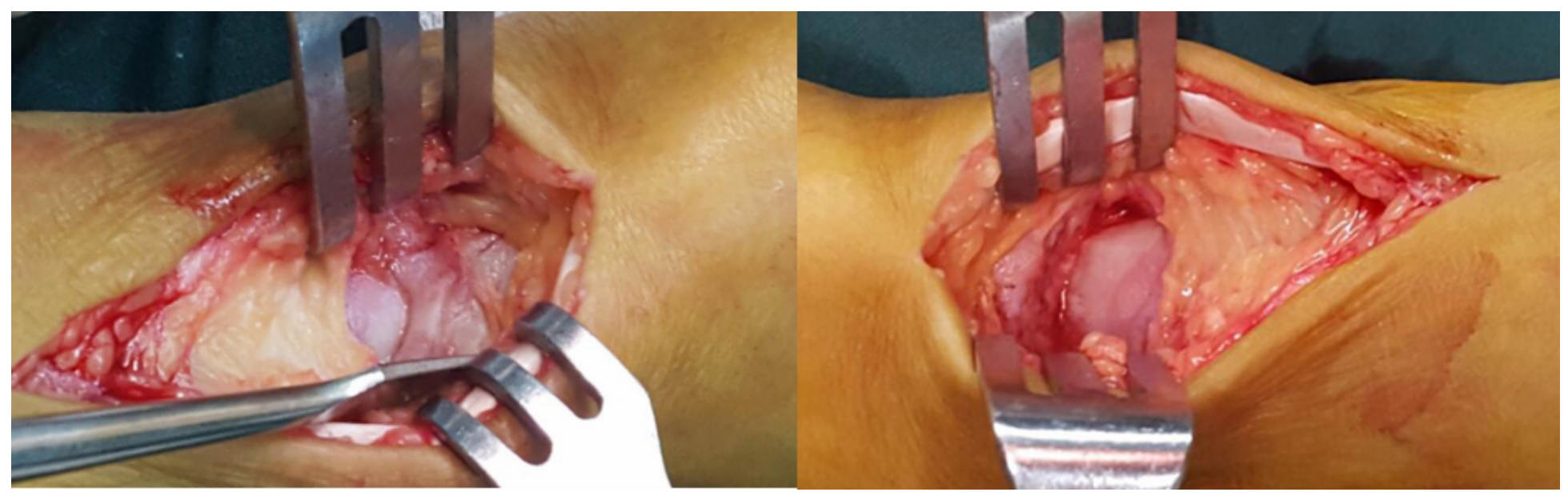

Figure 3

The osteophyte in front of the ankle joint was seen during the operation and completely removed.

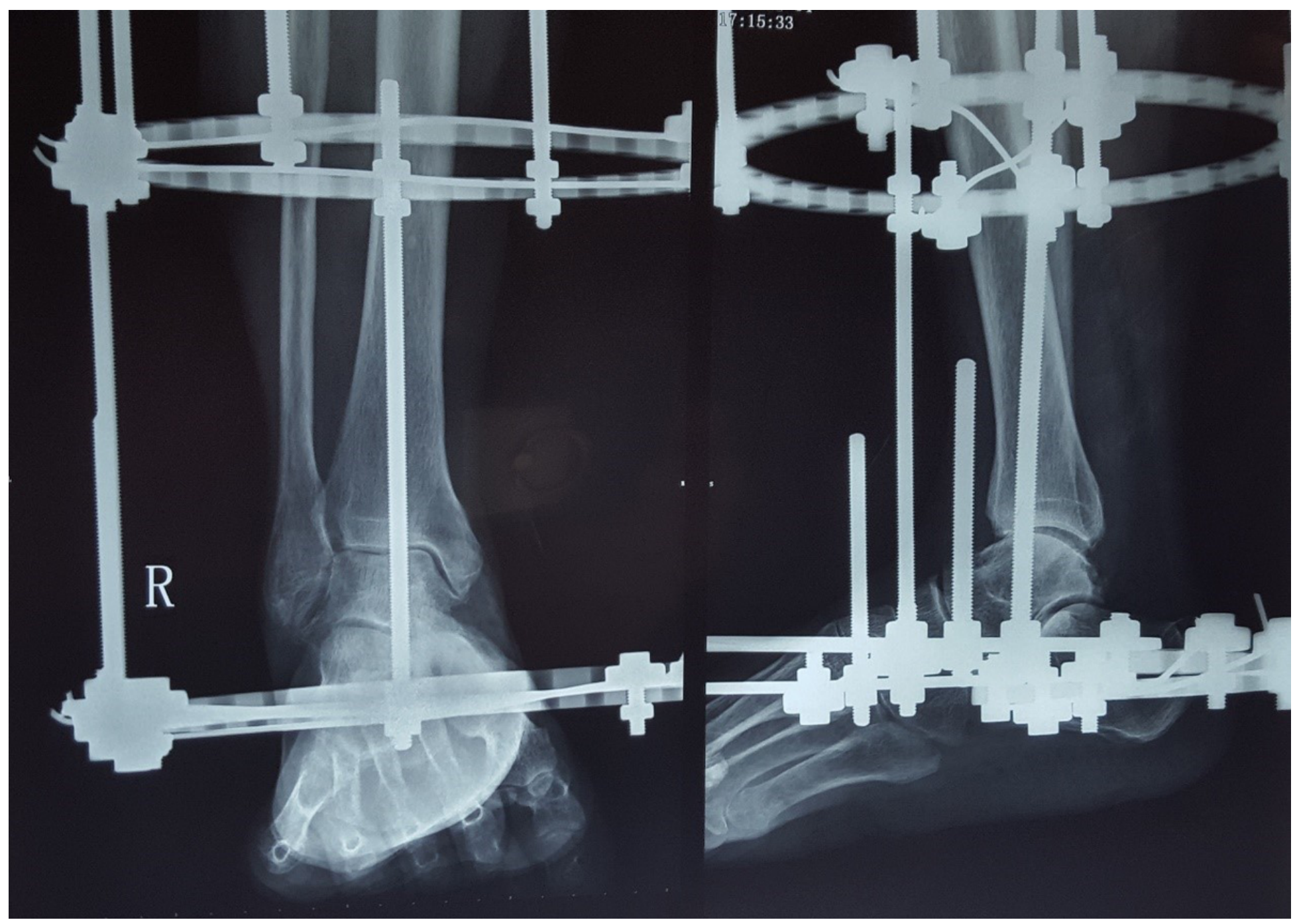

Figure 4

Seven days after operation, the ankle joint X-ray showed that the ankle joint space was slightly widened, and the osteophytes on the front of the tibia had been removed. 


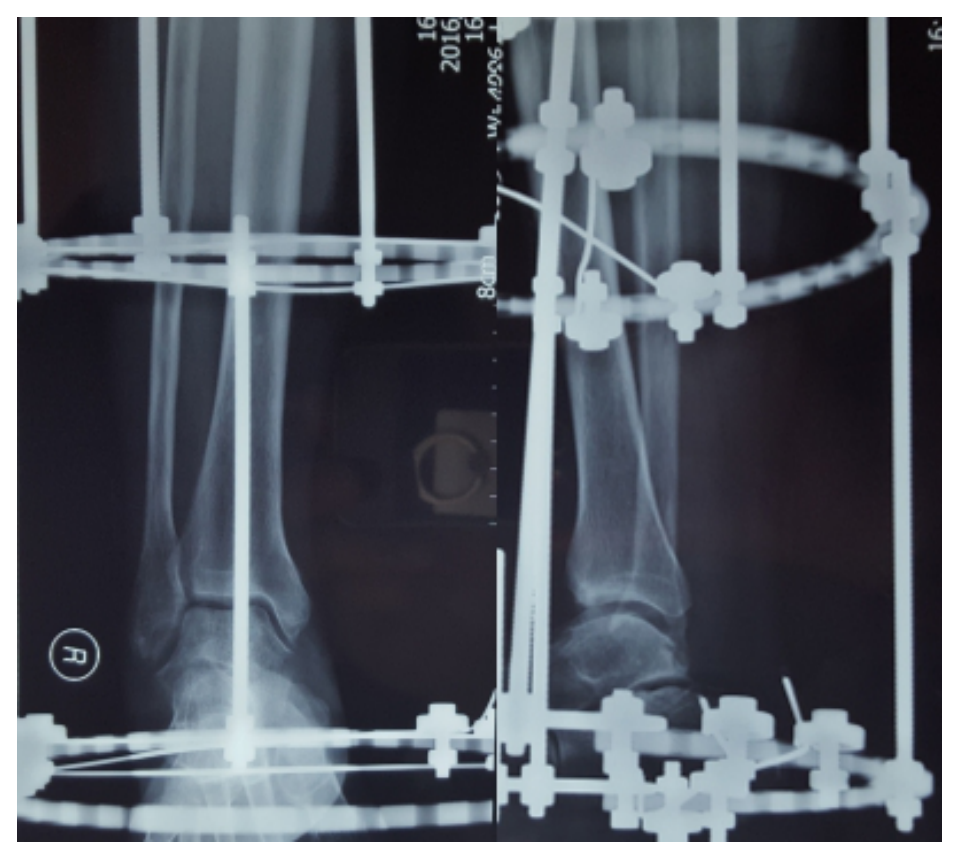

\section{Figure 5}

The anteroposterior and lateral X-ray of ankle joint 1.5 months after operation showed that the ankle joint space was almost normal, and the anterior lip of ankle joint did not proliferate.

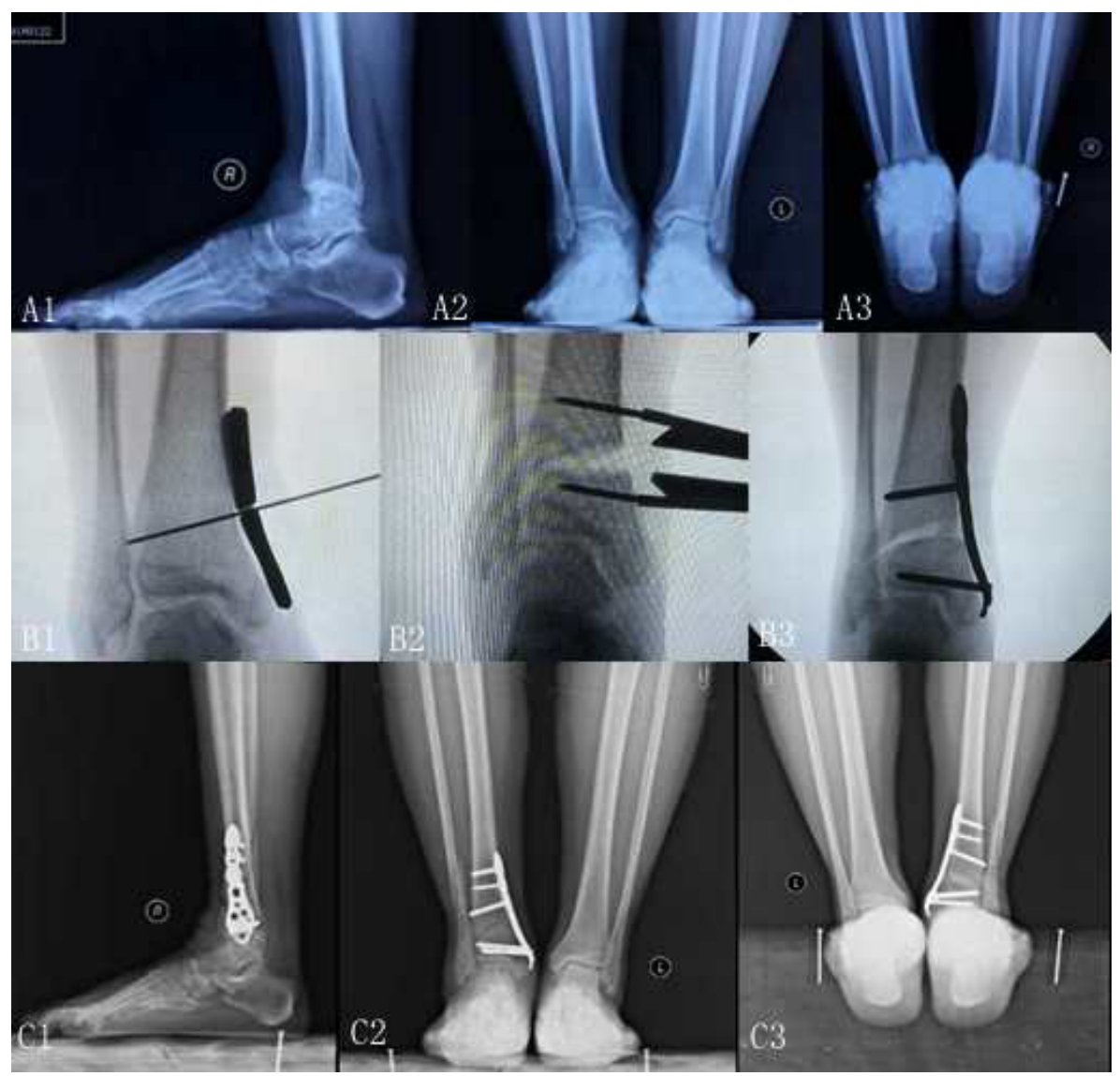

\section{Figure 6}

$\mathrm{A}$ is the preoperative lateral, anterior and calcaneal axis X-rays, showing ankle varus deformity and ankle arthritis; $\mathrm{B}$. intraoperative localization of supramalleolar osteotomy and internal fixation; C 2 months after operation, the force line 
of ankle joint was good and the ankle joint space was clear.

Page 15/15 